\title{
Bekanntheit des gesetzlichen Mindestlohns
}

\section{Ergebnisse repräsentativer Befragungen von Beschäftigten}

Seit dem 1. Januar 2015 gilt in Deutschland ein gesetzlicher Mindestlohn. Damit dieser seine Wirksamkeit entfalten kann, ist es nötig, dass die Vorschriften des Mindestlohngesetzes eingehalten werden und der Mindestlohn gezahlt wird. Die erfolgreiche Implementierung von Mindestlöhnen baut auf mehreren Säulen auf: eine ausreichende Information und Kenntnis über den Mindestlohn, Klarheit und Transparenz über die geltenden Regelungen, effektive Kontrollsysteme sowie Sanktionsmöglichkeiten (Low Pay Commission, 2017; Bosch et al., 2015; G.I.B., 2014; Benassi, 2011). Mit Blick auf die Kenntnis über den Mindestlohn hat das Markt- und Sozialforschungsinstitut Ipsos im Auftrag der Mindestlohnkommission drei repräsentative Beschäftigtenbefragungen durchgeführt (Ipsos 2020, 41 f., 57). Hierzu wurden im 4. Quartal 2018, im 2. Quartal 2019 und im 4. Quartal 2019 jeweils rund 2.000 abhängig Beschäftigte zur Existenz und Höhe des gesetzlichen Mindestlohns befragt. Die Interviews erfolgten als computergestützte, persönliche Befragungen (CAPI). ${ }^{1}$ Die zentralen Befunde aus diesen Befragungen werden im vorliegenden Beitrag präsentiert.

1 Die Interviews wurden im Rahmen von Mehrthemenumfragen (Omnibus-Befragungen), welche die Fragen verschiedener Projekte bündeln und durch ihre Themenmischung die Qualität der Erhebung sichern, durchgeführt. Die Auswahl der Befragten erfolgte zufällig nach dem Random-Route-Verfahren, bei dem ausgehend von einem zufällig ausgewählten Startpunkt der Interviewer bzw. die Interviewerin nach festgelegten Begehungsregeln die Befragungshaushalte ermittelt. Da dennoch nicht alle Bevölkerungsgruppen in gleichem Maße an solchen Befragungen teilnehmen, wurde die Struktur der ungewichteten Stichprobe durch eine Gewichtung an Eckwerte aus der amtlichen Statistik (Mikrozensus) angepasst. Die gewichteten Ergebnisse sind somit repräsentativ für die deutschsprachige Wohnbevölkerung in Privathaushalten ab 14 Jahren (vgl. ausführlich Ipsos, 2020).

Dr. Oliver Bruttel leitet die Geschäfts- und Informationsstelle für den Mindestlohn in Berlin.

Dr. Matthias Dütsch ist wissenschaftlicher Mitarbeiterin der Geschäfts- und Informationsstelle für den Mindestlohn.

\section{Kenntnis der Höhe des Mindestlohns}

Bei der ersten Befragung Ende des Jahres 2018 betrug die Höhe des Mindestlohns noch 8,84 Euro pro Stunde, bevor er dann zum 1. Januar 2019 auf 9,19 Euro angehoben wurde. Die Frage „Wissen Sie, ob es in Deutschland einen gesetzlichen Mindestlohn gibt, also einen Lohn, den Beschäftigte mindestens erhalten müssen?" haben über alle drei Befragungszeitpunkte hinweg jeweils rund $95 \%$ der Befragten bejaht. Zwar wissen somit fast alle Beschäftigten, dass es einen gesetzlichen Mindestlohn in Deutschland gibt, allerdings konnten jeweils nur rund $60 \%$ aller Befragten einen konkreten Wert für die Höhe des Mindestlohns angeben. Im Jahr 2018 kannten, wie in Tabelle 1 (blau unterlegt) zu ersehen ist, $18 \%$ der Befragten die genaue Höhe von 8,84 Euro. Anfang 2019 konnten $13 \%$ der Personen den damals seit wenigen Monaten geltenden angepassten Betrag korrekt benennen. Der prozentuale Anteil lag Ende des Jahres 2019 erneut bei $18 \%$. Zudem ist der Anteil derjenigen Personen gestiegen, die einen Mindestlohn oberhalb von 9,19 Euro genannt haben: Während dies Ende des Jahres 2018 noch $7 \%$ waren, stieg der Anteil im 2. Quartal 2019 auf $24 \%$ und im 4. Quartal 2019 auf $30 \%$. Die relativ geringe Kenntnis über die Mindestlohnhöhe bestätigt auch die qualitative Studie von Koch et al. (2020, 102 ff.). Demnach ist die exakte Höhe des Mindestlohns bei Betrieben, Betriebsräten und besonders ausgeprägt bei Beschäftigten kaum bekannt.

Es zeigt sich somit, dass vergleichsweise wenige Befragte die genaue Höhe des Mindestlohns benennen können. Legt man hingegen ein symmetrisches 50 -Cent-Intervall um den exakten Wert zugrunde, also 25 Cent unterhalb und 25 Cent oberhalb, dann erhöht sich der Anteil deutlich. In diesem Fall nennen zusätzlich zwischen $29 \%$ und $32 \%$ der Arbeitnehmerinnen und Arbeitnehmer einen Wert in dieser Bandbreite (vgl. Tabelle 2). In Summe kennt

(C) Der/die Autor(en) 2020. Open Access: Dieser Artikel wird unter der Creative Commons Namensnennung 4.0 International Lizenz (https:// creativecommons.org/licenses/by/4.0/deed.de) veröffentlicht.

Open Access wird durch die ZBW - Leibniz-Informationszentrum Wirtschaft gefördert.

* Der Artikel gibt ausschließlich die Meinung der Autoren und nicht notwendigerweise die der Mindestlohnkommission wieder. 
Tabelle 1

Bekanntheit der konkreten Höhe des Mindestlohns

in \% der Befragten

\begin{tabular}{lccc}
$\begin{array}{l}\text { Angaben zur Höhe des } \\
\text { Mindestlohns in Euro }\end{array}$ & $\begin{array}{c}\text { 4. Quartal } \\
2018\end{array}$ & $\begin{array}{c}\text { 2. Quartal } \\
2019\end{array}$ & $\begin{array}{c}\text { 4. Quartal } \\
2019\end{array}$ \\
\hline weniger als 8,50 & 4 & 2 & 0 \\
\hline 8,50 & 9 & 3 & 2 \\
\hline 8,51 bis 8,83 & 8 & 2 & 1 \\
\hline 8,84 & 18 & 4 & 1 \\
\hline 8,85 bis 9,18 & 13 & 13 & 9 \\
\hline 9,19 & 0 & 13 & 18 \\
\hline 9,20 bis 9,35 & 2 & 8 & 10 \\
\hline 9,36 bis 9,99 & 2 & 8 & 11 \\
\hline 10 bis 11,99 & 3 & 7 & 8 \\
\hline 12 und mehr & 0 & 1 & 1 \\
\hline Mindestlohnhöhe nicht & 37 & 36 & 34 \\
\hline bekannt/weiß nicht & 5 & 5 & 5 \\
\hline $\begin{array}{l}\text { Es gibt keinen Mindestlohn/ } \\
\text { weiß nicht }\end{array}$ & & &
\end{tabular}

Anmerkung: Die Frage lautete: „Wissen Sie vielleicht auch, wie hoch der gesetzliche Mindestlohn aktuell ist?"

Basis: Abhängig Beschäftigte im Alter von 18 bis 65 Jahren.

Quelle: Repräsentative Befragung von Ipsos im Auftrag der Mindestlohnkommission, eigene Berechnungen.

damit rund die Hälfte aller Beschäftigten die ungefähre Höhe des Mindestlohns. Beschäftigte mit einem Stundenverdienst von weniger als 11 Euro können sowohl die exakte Höhe des gesetzlichen Mindestlohns als auch die Bandbreite, in der er sich bewegt, weniger gut benennen als Beschäftigte mit höheren Stundenverdiensten. ${ }^{2}$ So gaben im 4. Quartal 2019 13\% der Niedriglohnbeziehenden die exakte Höhe des gesetzlichen Mindestlohns, weitere $28 \%$ Werte im 50-Cent-Intervall um den exakten Wert an. Insgesamt waren dies unter den Niedriglohnbeziehenden somit $41 \%$ im Vergleich zu $47 \%$ bei allen Beschäftigten. Dieser Befund ist insofern bemerkenswert, als zu erwarten gewesen wäre, dass der Mindestlohn als Referenzwert bei Niedriglohnverdienerinnen und -verdienern eine größere Rolle spielt als in höheren Einkommensgruppen.

\section{Unterschiede zwischen Beschäftigtengruppen}

Betrachtet man die Ergebnisse zur Bekanntheit des Mindestlohns differenziert nach verschiedenen Beschäf-

2 Die Höhe des eigenen Stundenlohns wurde durch folgende direkte Frage ermittelt: „Können Sie mir bitte sagen, wie viel Sie pro Stunde verdienen? Liegt ihr vereinbarter Bruttostundenlohn unter 11 Euro oder bei 11 Euro oder darüber?"
Tabelle 2

Kenntnis über die Höhe des Mindestlohns in einem 50-Cent-Intervall um den genauen Wert

in \% der Befragten

\begin{tabular}{|c|c|c|c|c|c|c|}
\hline & \multicolumn{3}{|c|}{ Beschäftigte insgesamt } & \multicolumn{3}{|c|}{$\begin{array}{l}\text { Beschäftigte mit Stunden- } \\
\text { Iöhnen unter } 11 \text { Euro }\end{array}$} \\
\hline & $\begin{array}{l}\text { Exakter } \\
\text { Wert }\end{array}$ & $\begin{array}{l}\text { 50-Cent- } \\
\text { Intervall } \\
\text { um exak- } \\
\text { ten Wert }\end{array}$ & Summe & $\begin{array}{l}\text { Exakter } \\
\text { Wert }\end{array}$ & $\begin{array}{l}\text { 50-Cent- } \\
\text { Intervall } \\
\text { um exak- } \\
\text { ten Wert }\end{array}$ & Summe \\
\hline \multicolumn{7}{|l|}{ 4. Quartal } \\
\hline 2018 & 18 & 32 & 50 & 15 & 27 & 42 \\
\hline \multicolumn{7}{|l|}{ 2. Quartal } \\
\hline 2019 & 13 & 31 & 44 & 9 & 27 & 36 \\
\hline $\begin{array}{l}\text { 4. Quartal } \\
2019\end{array}$ & 18 & 29 & 47 & 13 & 28 & 41 \\
\hline
\end{tabular}

Anmerkung: Die Frage lautete: „Wissen Sie vielleicht auch, wie hoch der gesetzliche Mindestlohn aktuell ist?"

Basis: Abhängig Beschäftigte im Alter von 18 bis 65 Jahren.

Quelle: Repräsentative Befragung von Ipsos im Auftrag der Mindestlohnkommission, eigene Berechnungen.

tigtengruppen, dann lassen sich im Durchschnitt - mit Ausnahme des Merkmals zum eigenen Verdienst der befragten Personen - kaum Unterschiede erkennen (vgl. Abbildung 1). Bezogen auf alle drei Wellen kennen $16 \%$ den exakten Wert des Mindestlohns, weitere $31 \%$ kennen den Wert innerhalb des 50-Cent-Intervalls um den exakten Wert. In Summe nennen somit $47 \%$ den exakten Wert oder einen Wert innerhalb des 50-Cent-Intervalls. Dabei gibt es kaum Unterschiede zwischen Frauen und Männern, West- und Ostdeutschen, unterschiedlichen Altersgruppen oder entlang des Merkmals zur Stellung im Beruf. Lediglich der Stundenlohn macht einen Unterschied: Beschäftigte mit einem Stundenlohn von weniger als 11 Euro sind deutlich seltener als Beschäftigte mit höheren Stundenlöhnen in der Lage, sowohl den exakten Wert als auch einen Wert im 50-Cent-Intervall zu benennen. ${ }^{3}$

Fazit

Die genaue Höhe des Mindestlohns ist nur vergleichsweise wenigen Beschäftigten bekannt. Dies gilt insbesondere für Beschäftigte im Niedriglohnbereich, für die der gesetzliche Mindestlohn eine besonders hohe Relevanz haben dürfte. Zudem scheint nach einer Erhöhung des Mindestlohns dessen neuer Wert erst mit einer zeitlichen

3 Die zentralen Befunde, dass soziodemografische und tätigkeitsspezifische Merkmale eine lediglich geringe, der Verdienst der Befragten hingegen eine vergleichsweise große Bedeutung hinsichtlich der Bekanntheit des Mindestlohns haben, zeigen sich auch in Regressionsschätzungen, in denen die entsprechenden Koeffizienten beim Stundenverdienst hochsignifikant sind. 


\section{Abbildung 1}

Gruppenspezifische Unterschiede bezüglich der Bekanntheit des Mindestlohns

in \% der Befragten

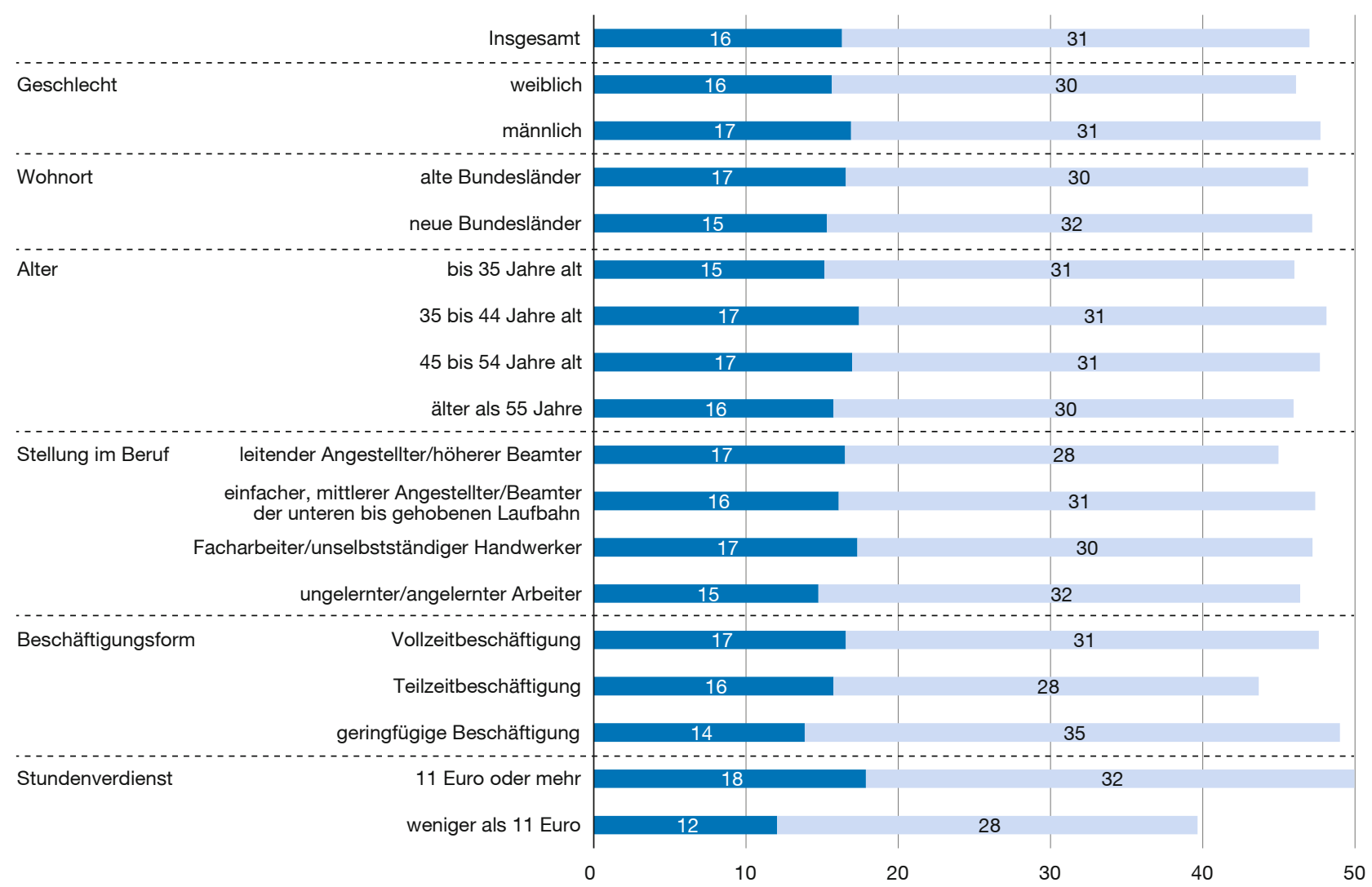

- Bekanntheit des exakten Werts des Mindestlohns

Bekanntheit des Mindestlohns im 50-Cent-Intervall

Basis: Abhängig Beschäftigte im Alter von 18 bis 65 Jahren (gepoolt über alle drei Wellen, $N=6.083$ ).

Quelle: Repräsentative Befragung von Ipsos im Auftrag der Mindestlohnkommission, eigene Berechnungen.

Verzögerung den Beschäftigten bewusst zu sein. Um die Bekanntheit der exakten Mindestlohnhöhe zu steigern, hat die Mindestlohnkommission in ihrem aktuellen Bericht (Mindestlohnkommission, 2020, 57) darauf hingewiesen, dass es wichtig sei, dass das Bundesministerium für Arbeit und Soziales, die Geschäfts- und Informationsstelle für den Mindestlohn und die Sozialpartner regelmäßig über die aktuelle Höhe des Mindestlohns informieren.

\section{Literatur}

Benassi, C. (2011), The implementation of minimum wage: Challenges and creative solutions, Global Labour University Working Paper, 12, International Labour Office.

Bosch, G., K. Jaehrling und C. Weinkopf (2015), Gesetzlicher Mindestlohn in der Praxis - Bedingungen für eine erfolgreiche Umsetzung, WISO direkt, 6/2015, Friedrich-Ebert-Stiftung.
G.I.B. (Gesellschaft für innovative Beschäftigungsförderung) (2014), Umsetzung und Kontrolle von Mindestlöhnen. Europäische Erfahrungen und was Deutschland von innen lernen kann, Arbeitspapiere, 49, November.

Ipsos (2020), Bekanntheit des gesetzlichen Mindestlohns. Ergebnisse von drei repräsentativen Befragungen von Beschäftigten, Studie im Auftrag der Mindestlohnkommission, https://www.mindestlohn-kommission.de/DE/Forschung/Projekte/pdf/Bericht-Umfrage-Bekanntheit-Mindestlohn.html?nn=8711200 (3. August 2020).

Koch, A., A. Kirchmann, M. Reiner, T. Scheu, A. Zühlke und H. Bonin (2020), Verhaltensmuster von Betrieben und Beschäftigten im Kontext des gesetzlichen Mindestlohns, Studie im Auftrag der Mindestlohnkommission, Institut für Angewandte Wirtschaftsforschung, Forschungsinstitut zur Zukunft der Arbeit und SOKO Institut für Sozialforschung und Kommunikation, https://www.mindestlohn-kommission.de/DE/Forschung/Projekte/pdf/Bericht-Verhalten-BetriebeBeschaeftigte-Mindestlohn.html?nn=8711200 (3. August 2020).

Low Pay Commission (2017), Non-compliance and enforcement of the national minimum wage. 